\title{
Real-time tumor tracking with an artificial neural networks-based method: A feasibility study
}

\author{
Matteo Seregni ${ }^{a, *}$, Andrea Pella ${ }^{a}$, Marco Riboldi ${ }^{a, b}$, Roberto Orecchia ${ }^{\text {c,d,e }}$, \\ Pietro Cerveri ${ }^{a}$, Guido Baroni ${ }^{a, b}$
}

a Department of Bioengineering - TBMLab, Politecnico di Milano, P.zza Leonardo da Vinci 32, I-20133 Milano, Italy
b Bioengineering Unit, Centro Nazionale di Adroterapia Oncologica, Strada privata Campeggi, I-27100 Pavia, Italy
c Division of Radiation Oncology, European Institute of Oncology, Via Ripamonti 435, I-20141 Milano, Italy
${ }^{d}$ Clinical Area, Centro Nazionale di Adroterapia Oncologica, Strada privata Campeggi, I-27100 Pavia, Italy
e Department of Science and Biomedical Technologies, Universita di Milano, Milano, Italy

Received 3 August 2011; received in revised form 21 October 2011; accepted 16 November 2011

Available online 29 December 2011

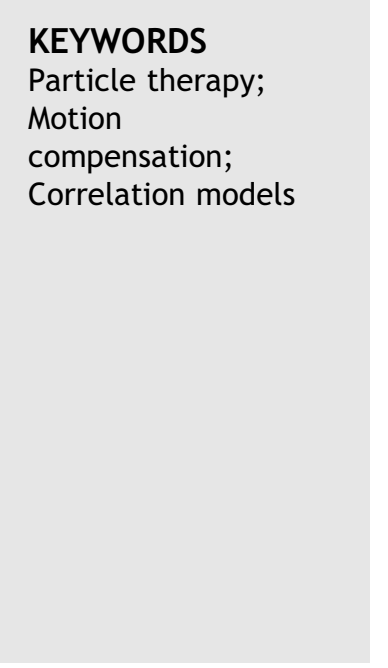

\begin{abstract}
The purpose of this study was to develop and assess the performance of a tumor tracking method designed for application in radiation therapy. This motion compensation strategy is currently applied clinically only in conventional photon radiotherapy but not in particle therapy, as greater accuracy in dose delivery is required.

We proposed a tracking method that exploits artificial neural networks to estimate the internal tumor trajectory as a function of external surrogate signals. The developed algorithm was tested by means of a retrospective clinical data analysis in 20 patients, who were treated with state of the art infra-red motion tracking for photon radiotherapy, which is used as a benchmark. Integration into a hardware platform for motion tracking in particle therapy was performed and then tested on a moving phantom, specifically developed for this purpose.

Clinical data show that a median tracking error reduction up to $0.7 \mathrm{~mm}$ can be achieved with respect to state of the art technologies. The phantom study demonstrates that a real-time tumor position estimation is feasible when the external signals are acquired at $60 \mathrm{~Hz}$.

The results of this work show that neural networks can be considered a valuable tool for the implementation of high accuracy real-time tumor tracking methodologies.

(c) 2011 Associazione Italiana di Fisica Medica. Published by Elsevier Ltd. All rights reserved.
\end{abstract}

\footnotetext{
${ }^{*}$ Corresponding author.

E-mail address: matteo.seregni@mail.polimi.it (M. Seregni).
}

1120-1797/\$ - see front matter @ 2011 Associazione Italiana di Fisica Medica. Published by Elsevier Ltd. All rights reserved. doi:10.1016/j.ejmp.2011.11.005 


\section{Introduction}

In radiation therapy, ionizing radiation is used to damage malignant cells as part of a treatment course to cure cancer. Radiation therapy is actually used in approximately $60 \%$ of patients as curative, adjuvant or palliative treatment strategy. The fundamental principle of radiation therapy is to cause the highest possible damage to the neoplastic target while, at the same time, sparing surrounding healthy structures. However, tumors placed in the thoracic and abdominal areas are often influenced by intra-fractional organ motion, caused by physiological processes such as breathing and heartbeat [1-3]. In these cases, uncertainties in target localization force to expand the geometric margins around the clinical target volume $[4,5]$. The end result is an increase in the volume of healthy tissues which is irradiated, thus reducing the ballistic potentialities and the geometrical selectivity of the treatment. As a consequence, motion monitoring assumes a very significant role in radiation therapy.

External motion tracking is typically performed for patient set-up and respiratory motion monitoring [6]. Moreover, it can also be exploited as a surrogate of the internal motion, either for 4DCT reconstruction [7] or when the treatment is delivered by means of motion mitigation techniques, such as beam gating and beam tracking, which will be specifically described later in this section. The displacement of the external body surface is generally detected relying on infra-red devices [8]. For example the Real-Time Position Management (RPM, Varian Medical Systems, Palo Alto, CA) system is able to localize a single object placed on the patient's abdomen [9]. However, in order to detect the motion in all its complexity, multiple passive markers can be placed on the thorax and/or on the abdomen of the patient. Alternative solutions that do not require external markers, whose placement is time consuming and requires very high reproducibility, have been developed relying on surface imaging [10].

Also the internal motion can be directly monitored in radiation therapy [11]. For this purpose, a metallic marker is typically implanted inside or near the tumor and its position is tracked in real-time by means of fluoroscopic imaging. In this case, Real Time Radiation Therapy (RTRT) [12] can be implemented by continuously detecting the internal tumor position. Results show that targets moving at a speed up to $40 \mathrm{~mm} / \mathrm{s}$ can be tracked at a frequency of 30 images per second with an accuracy better than $1.5 \mathrm{~mm}$ [13]. However, the main drawback of this technique consist in the amount of non therapeutic dose that is delivered to the patients as a consequence of the fluoroscopic acquisition that is performed during the entire duration of the treatment.

In order to achieve an accurate dose delivery even on moving targets, internal motion monitoring is generally combined to motion mitigation techniques, whose aim is to compensate for intra-fractional organ motion [14]. Among these techniques, the breath hold consists of a forced [15] or voluntary $[16,17]$ suspension of respiration during irradiation; conversely, respiratory gating limits irradiation to a pre-defined respiratory phase identified by a respiratory related signal, which is provided by external surrogates
$[18,19]$. Another possible approach to motion mitigation is tumor tracking, which implies the dynamic steering of the therapeutic beam in order to follow in real-time the target motion along its trajectory. Tumor tracking shows significant advantages with respect to both breath hold and gating: in particular, it allows the patient to breath freely for the entire duration of the treatment, without requiring any suspension of the respiratory cycle, which may result difficult in subjects suffering from respiratory impairment. Moreover, since tumor tracking allows the dose to be delivered almost continuously, a high duty cycle can be achieved, thus reducing the total treatment duration with respect to respiratory gating and breath hold.

At present, tumor tracking is implemented clinically in the CyberKnife system (Accuray Inc., Sunnyvale, CA) equipped with the Synchrony module [20,21]. This device provides real-time tumor tracking relying on the correlation between internal target and external marker motion. It works as follows: before the irradiation the tumor position is determined at multiple distinct time points in different breathing phases with two orthogonal X-ray acquisitions. Then a linear, quadratic or constrained fourth order polynomial correspondence model is generated to relate tumor position to the three external markers. During treatment, this correlation model is used to estimate the internal tumor position as a function of external markers motion. Additional X-ray images at limited temporal frequency are acquired during treatment, in order to check and update the correlation model when needed. Since Synchrony uses three optical external markers, the system builds a correlation model for each marker; then the outputs of each model are averaged to obtain a unique estimation. Moreover, to take into account communication latencies and the inertia of the manipulator ( $\approx 115 \mathrm{~ms}$ overall), a time predictor is used to compensate for these delays. It has been reported that in real patient treatments the accuracy of the Cyberknife equipped with Synchrony RTS ranges from $0.2 \mathrm{~mm}$ to $1.9 \mathrm{~mm}$ in superior-inferior direction, from $0.1 \mathrm{~mm}$ to $1.9 \mathrm{~mm}$ in left-right direction and from $0.2 \mathrm{~mm}$ to $2.5 \mathrm{~mm}$ in anterior-posterior direction [23]. Recent studies $[22,23]$ have shown that its accuracy in tumor tracking is mostly dependent on uncertainties in the internal/external correlation model, rather than on the prediction.

Starting from these findings, we recently performed a comparative study to assess the accuracy of different correlation algorithms for tumor tracking [24]. This was conducted on a population of twenty patients who received extra-cranial radiotherapy with real-time compensation of tumor motion by means of the Synchrony RTS. A linear/ quadratic model was compared with more complex strategies, including artificial neural networks and fuzzy logic: results showed that complex methods provide better accuracy in tumor tracking with respect to a polynomial correlation model, particularly in irregularly breathing patients. However, the algorithms and the method proposed in this study are meant for offline data analysis. As consequence, they cannot be integrated into a motion monitoring system and exploited for online data acquisition and processing.

Starting from the results of our previous work [24], we propose a study with the following goals: 
- Development of a correlation algorithm based on artificial neural networks, as this method was reported to provide higher accuracy in tumor position estimation with respect to polynomial correlation. This algorithm was designed to be integrated into an optical localization system used for external motion monitoring and to be able to acquire and processes data in real-time.

- Validation of the developed algorithm on a clinical dataset coming from a group of patients treated with Synchrony.

- Assessment of the developed algorithm by means of tests conducted during online acquisitions performed on a moving phantom, which was specifically developed for this purpose.

The expected result of this work is a tumor tracking method comparable to the Synchrony RTS in terms of tracking accuracy and integrated in the frame of an optical localization system used for external motion monitoring in radiation therapy, expanding in this way the results presented in reference 24. Moreover, this expected outcome will be of relevance non only in the field of conventional radiotherapy (i.e. in treatments delivered with photons beams), but also in particle therapy, where dose is delivered by means of charged particles (mainly protons and carbon ions) [25] and the technical feasibility of a dynamic tumor tracking system has already been proved $[26,27]$ but still not applied in clinics.

\section{Materials and methods}

We developed a tumor tracking method based on artificial neural networks (ANNs), which are non-linear computational models able to face black-box problems learning from examples.

In our work, ANNs have been implemented in $\mathrm{C} / \mathrm{C}++$ language and exploited to estimate internal target motion as a function of external surrogate signals, given by the position of three reflective markers.

\section{General workflow for real-time tumor tracking}

The developed method was designed to be applied in the frame of the workflow for real-time tumor tracking based on internal/external correlation, which we describe hereafter (Fig. 1):

i. at the beginning of the treatment, a training dataset is collected. This set must contain samples of the external signals, i.e. the 3D position of the three markers, coupled with simultaneous samples of the 3D tumor position (internal signal). The number of this samples is limited by the constraints due to imaging dose, as X-ray imaging is typically applied to localize the tumor. For this reason, the workflow includes spline interpolation at this stage in order to obtain a training dataset containing 500 distinct examples.

ii. Once the training dataset has been collected, three different neural networks, one for each of the external markers, are trained with a back-propagation algorithm based on gradient descent. We selected a feed- forward double-layer architecture featuring a hidden layer of 10 units and a hyperbolic tangent activation function, which is applied to both the hidden and the output layer.

Termination criteria where defined so that the training algorithm is stopped when one of the two following condition is verified:

- Number of iteration $>100$. During a single iteration, all examples contained in the training set are used to adjust the inter-synaptic weights. When the 100th iteration is completed, this condition is considered as verified.

- RMS error $<0.01 \mathrm{~mm}$. The RMS error measures the distance between simulated outputs and ground truth tumor positions. It is defined as follows:

$R M S=\sqrt{M S E}=\sqrt{\frac{1}{p} \sum_{\mu=1}^{P} \sum_{i=1}^{N}\left(U_{d i}^{\mu}-U_{i}^{\mu}\right)^{2}}$

where $U_{d i}$ is the desired output of the $i$-th unit of the output layer and $U_{i}$ is the simulated output of the same unit. This error is calculated for each units of the output layer $(i=$ $1 \ldots N)$ and for each input/output example contined in the training set $(\mu=1 \ldots P)$.

Both these criteria were defined in order to avoid the effects due to overfitting, which may result in poor performance when simulating data that are not included in the training set. Hence these values represent a compromise between the optimization of training performance and the ability to generalize unknown input data.

iii. Trained ANNs are exploited for the estimation of the target position as a function of each sample of the external signal. Since three independent estimations are obtained (one for each network), a unique output is calculated by averaging the outputs of the three ANNs.

iv. To take into account any modification in the correlation between the external and internal signals [22], the proposed algorithm includes ANN retraining when necessary. The retraining process was implemented as a threshold based procedure depending on the error on tumor position estimation; this information is obtained by comparing estimated versus ground truth tumor position, being this latter provided by periodic X-ray control images. The estimation error is calculated for each network: if it exceeds a user-defined threshold, the neural network is retrained using a training set which is updated by including the most recent input/ output data and discarding the oldest one (First In First Out logic, FIFO).

\section{CyberKnife data analysis}

The performance of the algorithm was quantified, in terms of 3D targeting errors, on clinical data extracted from a database of 130 patients who received stereotactic body radiotherapy with Cyberknife equipped with the Synchrony tracking module. These data, which are made available by 


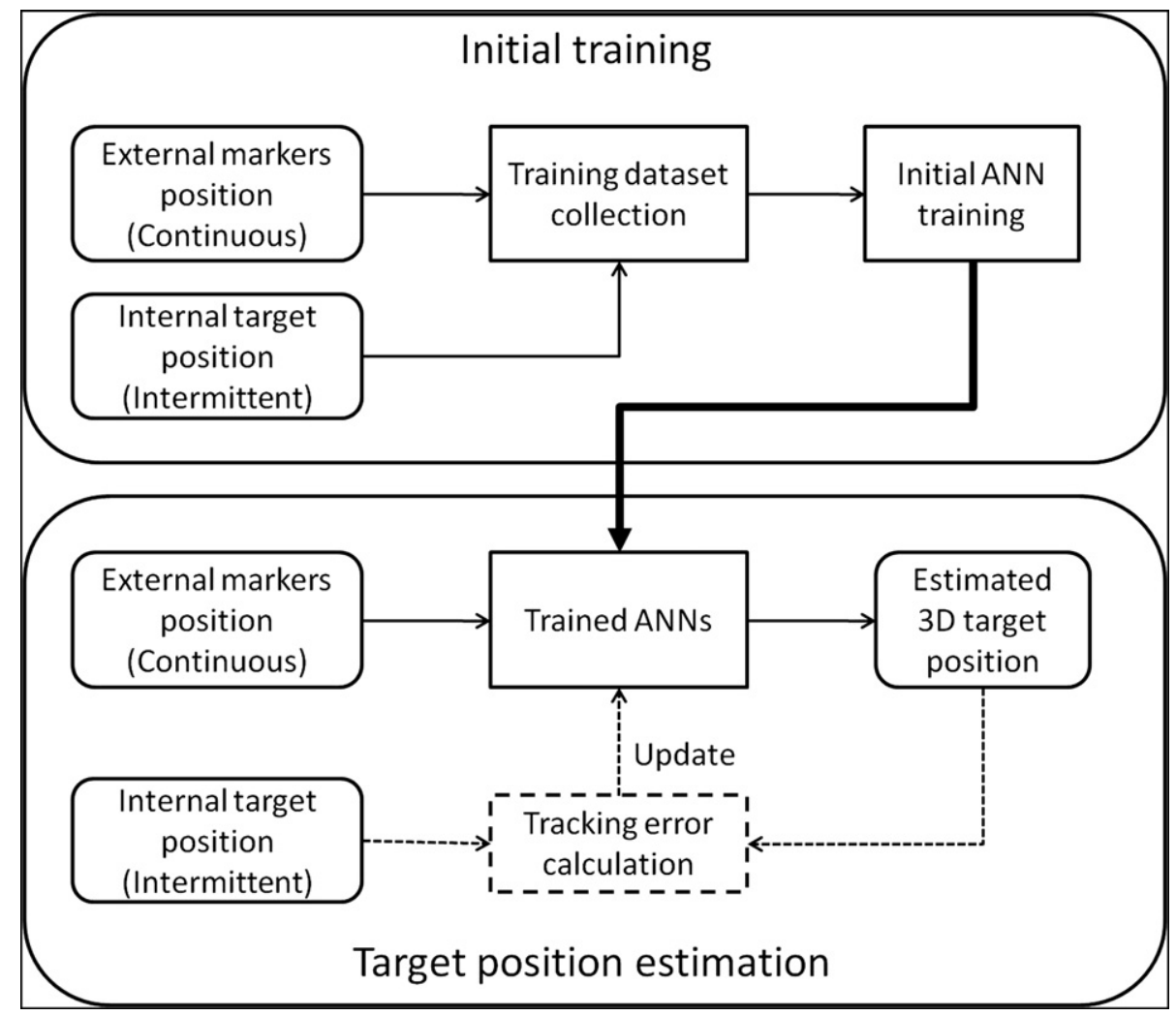

Figure 1 Flowchart of the proposed tumor tracking algorithm.

the Georgetown University Medical Center (Washington, DC), consist of a set of log files containing, for each treatment session, the following information:

- The position of three external markers, placed on tight fitting vest, acquired with an infra-red tracking system during the entire treatment duration at a frequency of approximately $25 \mathrm{~Hz}$.

- The position of the target, obtained through orthogonal $X$-ray images where the clips implanted into or near the tumor are localized. These X-ray images are acquired (with an average period of $25.7 \mathrm{~s}$ in the considered dataset) in order to build the external/internal correlation model before irradiation. Additional control images are acquired during treatment (average period $\approx 66.1 \mathrm{~s}$ ) to check and update the model as needed.

Both the external and the internal signals share a common temporal basis so that it is possible to obtain simultaneous input/output data to be used for ANN training.

Twenty patients among the 130 available were selected and divided into two different groups [24]. The first one contains 10 worst cases, defined as those fractions exhibiting the largest average tracking error (calculated from the Synchrony log files), whereas the second group, referred to as control group, contains ten patients randomly selected among the remaining population.

For each of these patients we applied the developed algorithm in the frame of a treatment simulation process, considering as external signal the position of the three markers placed on the vest and, as internal signal, the target position localized through intermittent $\mathrm{X}$-ray imaging.

The simulation procedure was designed according to the four steps described above in the general workflow explanation: at the beginning, the algorithm collects an initial training dataset containing the same X-ray acquisitions used by the Synchrony module to build the correspondence model, then the three ANNs are trained and used to estimate the target position as a function of the external signal.

When a control image is available, the estimation obtained by each network is compared to the actual target position according to the following figure $E_{i}$ :

$$
\begin{aligned}
& S_{i}=\left[S_{x, i} S_{y, i} S_{z, i}\right] ; P=\left[P_{x} P_{y} P_{z}\right] ; \forall i=1 \ldots 3 \\
& E_{i}=\max \left(\left|S_{x, i}-P_{x}\right|,\left|S_{y, i}-P_{y}\right|,\left|S_{z, i}-P_{z}\right|\right)
\end{aligned}
$$

where $S_{i}$ is the target position estimated by the $i$-th network and $\boldsymbol{P}$ is the ground truth target position. Subsequently, the training dataset is updated with a FIFO approach.

We defined two threshold values ( $\alpha$ and $\beta$ ) both expressed in millimetres and referred to as retrain and rebuild threshold, respectively. The values of these thresholds are user-defined but $\beta$ is intended to be larger than (or equal to) $\alpha$ : we set $\alpha=1 \mathrm{~mm}$ and $\beta=2 \mathrm{~mm}$ in our implementation.

According to the value of $E_{\mathrm{i}}$, the algorithm develops as follows: 
- If $E_{\mathrm{i}} \leq \alpha$, the $\mathrm{i}$-th network is not retrained.

- If $\alpha<E_{\mathrm{i}} \leq \beta$, the $\mathrm{i}$-th network is retrained starting from current inter-synaptic weights.

- If $E_{\mathrm{i}}>\beta$, then the $\mathrm{i}$-th network is retrained starting from randomly initialized inter-synaptic weights. In these cases, a significant variation in the internal/ external correspondence model is expected due to the large measured tumor tracking errors. Hence, current inter-synaptic weights are discarded in order to have a quicker adaptation to the new condition.

At the end of the simulation, the performance of the tracking algorithm was quantified in MatLab (The MathWorks Inc., Natick, MA) by evaluating, for each control image, the 3D targeting error (TE), which is the distance between the estimated and the ground truth target position.

The same method was applied to quantify the 3DTEs relative to the Synchrony module and results were used as benchmark to understand the benefits of the new algorithm.

\section{Real-time implementation}

In order to test the real-time implementation of the developed algorithm and to check the integration into a hardware platform dedicated to radiation therapy applications, we performed dedicated experimental acquisitions. We used a home-designed and homemanufactured moving phantom specifically developed for respiratory motion simulation, consisting of the following main components (Fig. 2):

- "Rib Cage": The structure of the rib cage is simulated with three moving elements, two laterals and a central one. These elements are constrained to pivot at the top so that they can rotate outward and inward. Three markers are placed on the rib cage and optically localized to capture the body surface motion.

- Piston: The piston is a cylindrical metal rod whose bottom end is fixed to a wooden board positioned inside the rib cage. Its vertical motion forces both the "rib cage" and the "target" to move, as explained below.

- "Target": the target to be irradiated is simulated using a small element connected with an elastic band and two springs to the moving piston and to the support structure of the phantom. One marker can be positioned on this element to simulate the internal signal.

The phantom is manually operated through a pulley (mounted on its top) that has an elliptic cross section and whose rotation axis is positioned eccentrically (Fig. 3). In this way, an ideally continuous rotation of the pulley is converted into a vertical harmonic motion of the piston,

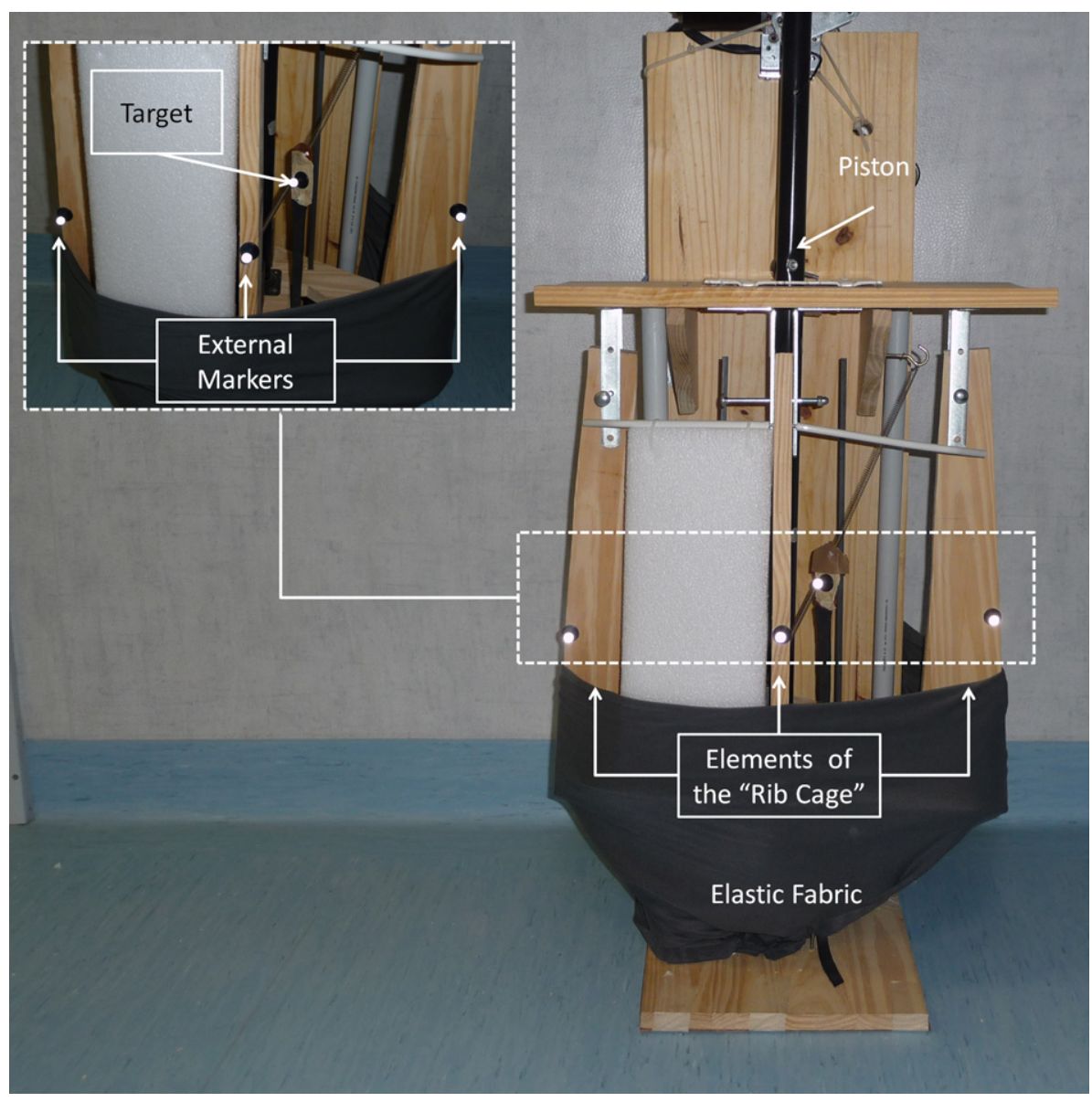

Figure 2 Moving phantom for respiratory motion simulation, with indications of its main components. 


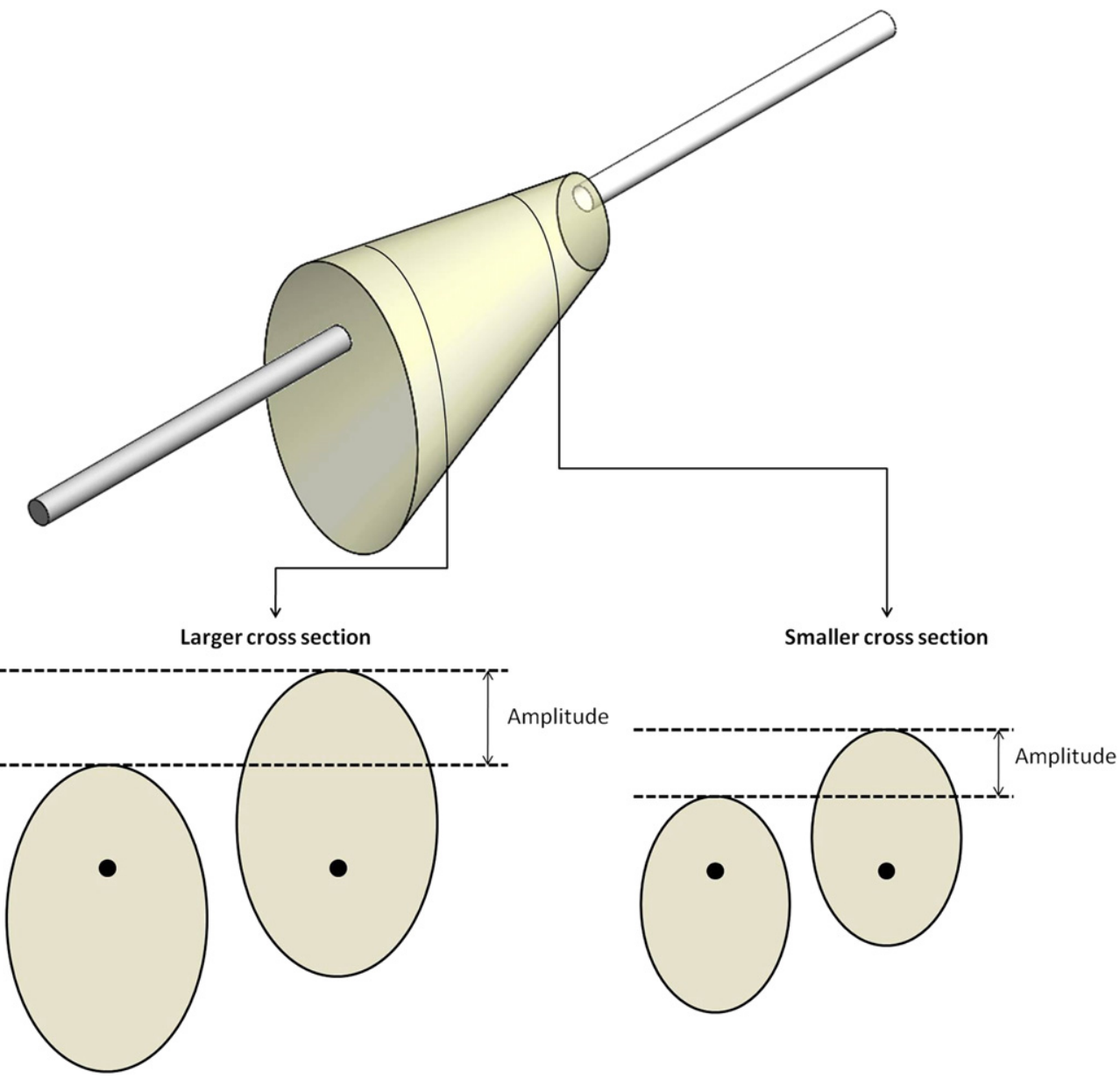

Figure 3 Schematic drawing of the pulley. How the motion amplitude changes as a function of the pulley's cross section is explained graphically.

which is connected to the pulley with a belt. Moreover, the pulley features a variable cross section across its length, so that the amplitude of vertical motion can be dynamically changed during the experiment, from approximately $5 \mathrm{~cm}$ to $11 \mathrm{~cm}$ (peak to peak). Frequency of motion is selected by the operator, who can apply the desired rotation speed.

The respiratory motion is simulated as follows:

- "Inspiration": when the piston moves down (by gravity), the elements of the rib cage rotate outward. At the same time the target is pulled down by the spring connected to the piston.

- "Expiration": when the piston is pulled up by the belt, the elements of the rib cage rotate inward as they are wrapped with a strip of elastic fabric or with an elastic rope. In the meantime the target returns to its original position.

Thanks to the springs configuration, the trajectory followed by the target during its movement is characterized by hysteresis, as observed for real tumor lesions [28]. It should be noted, however, that the main purpose of the phantom is not to exactly reproduce a physiological respiratory motion, but only to simulate internal and external signals with amplitudes and frequencies comparable to those observed in real subject, allowing us to validate internal/external correlation algorithms.

In order to perform online acquisitions using the moving phantom, we integrated the developed correlation algorithm into the optical localization system SMART-D (BTS Bioengineering S.p.A., Italy).

This system uses at least three TV cameras (TVC) to localize and track markers or laser spots with a manufacturer specification for accuracy of $0.2 \mathrm{~mm}$ in a working volume of $3 \times 2 \times 2 \mathrm{~m}$. The SMART-D is the main component of the Optical Tracking System (OTS) installed in the treatment rooms at CNAO (Centro Nazionale di Adroterpia Oncologica, Pavia, Italy) and will be exploited for initial patient alignment and monitoring during irradiation.

The integration of the correlation algorithm took advantage of a software provided with the SMART-D system, whose main function is to read the $3 D$ position of every point localized by the TVCs and to make it available to the user in real-time, i.e. at the acquisition frequency $(60 \mathrm{~Hz})$. Hence, during online acquisitions performed with the moving phantom, the spatial position of the three external markers that simulate the external signal, as well as the position of the fourth marker representing the target to be tracked), is available at the highest system sample rate.

In order to correctly identify the marker simulating the target and to provide a consistent input to the ANNs, an 
automatic labeling of the four markers is required at every acquired frame. For this reason, a dedicated algorithm was developed exploiting the a priori known distribution of the markers along the left-right direction of the phantom.

At each experimental phantom acquisition, the initial training dataset was collected for ANN training. In order to mimic episodic imaging, the 3D position of the marker representing the target, even if continuously available, was read only at discrete time points. We decided to collect an initial training dataset formed by 15 input/output data acquired at 150 frames distance $(2.5 \mathrm{~s}$ at the nominal acquisition frequency). Once all training data are available and the interpolation to reach 500 corresponding samples is completed, the algorithm proceeds with the initial training of the ANNs. In a second stage, the target position is estimated in correspondence of each acquired frame and checked intermittently with a period of about $16.5 \mathrm{~s}$ (once every 1000 acquired frames). The retraining strategy was implemented exactly as previously described for the simulation of Cyberknife treatments: the values of $\alpha$ and $\beta$ thresholds were set to $0.1 \mathrm{~mm}$ and $1 \mathrm{~mm}$, respectively.

Three experimental acquisitions on the moving phantom were performed according to the following protocol:

i. at the beginning of the acquisition, the phantom was moved approximately from maximum inspiration (piston pushed fully down) to maximum expiration position (piston pulled fully up). In this way the initial training data set is likely to be spread on the entire range of motion of the phantom.

ii. Afterwards, the pulley was operated to obtain breathing cycles at fixed amplitude and with a period of about $3 \mathrm{~s}$.

iii. During the acquisition, in order to simulate irregular breathing, the motion amplitude and frequency were dynamically varied.

The duration of these acquisitions was about 3 min each (from $173 \mathrm{~s}$ to $210 \mathrm{~s}$ ), whose first $40 \mathrm{~s}$ (approximately) were dedicated to the initial data collection and ANNs training.

During every acquisition, the software generates a log file which contains all the real-time estimations of the target position calculated by the algorithm and the actual target position acquired simultaneously by the SMART-D system. In addition, the frame number is recorded in correspondence to each estimation for offline analysis.

The real-time capability of the implemented application in estimating the target position was investigated by examining the sequence of the logged frames numbers: the dropping of one or more frames accounted for a missing real-time target position estimation.

The continuous availability of the correct position of the target at every frame allowed us to calculate 3DTE at each recorded frame.

\section{Results}

\section{CyberKnife data analysis}

3D targeting errors for the developed ANN-based method and for the Synchrony tracking system were calculated.
Overall median value, interquartile range and 95th percentile for the control and worst groups are reported in Table 1.

The cumulative probability distribution functions (PDFs) of the 3D targeting errors for both the control and worst groups are shown in Fig. 4.

A non parametric statistical analysis (Wilcoxon ranksum test) was applied to control and worst cases and it returned $p$-values equal to $2.1066 \times 10-5$ and 0.0012 respectively, thus proving a statistically significant error reduction for the ANN-based method relative to the Synchrony module.

In conclusion, the proposed method allowed an average 3D targeting reduction (with respect to Synchrony) of $17.84 \%$ for the control cases and $23.15 \%$ for the worst cases.

\section{Real-time implementation}

The log file analysis of the phantom experimental acquisitions showed that tumor position estimation took place consistently in real-time, except during the retraining of one or more ANNs. In these cases, the retrain procedure took, on average, a period of about 34 frames, corresponding to $0.57 \mathrm{~s}$ at the nominal acquisition frequency $(60 \mathrm{~Hz})$.

Table 2 reports the size of amplitude and period variability of the phantom respiratory signal due to simulated breathing irregularities.

In order to quantify the tracking accuracy in the three experimental acquisitions, 3D targeting errors are computed in correspondence to each acquired frame. This is possible since the actual position of the target, although considered intermittently by the algorithm, is continuously detected by the SMART-D system. Table 3 reports the median \pm interquartile range and 95th percentile of the 3DTEs which were continuously calculated as the distance between the estimated and the actual position of the marked target in the phantom.

As an example, Fig. 5 depicts a detail of the traces recorded during acquisition number 1 .

In this figure, it is possible to observe the effects of frequency and amplitude variations for irregular breathing simulation. Also, the temporary suspensions of the target position estimation due to the neural networks retraining (blank trace) can be noted.

\section{Discussion}

We developed a real-time tumor tracking method based on artificial neural networks, able to estimate the internal

Table 1 Statistical parameters of the 3D targeting errors recorded in the control and worst groups.

\begin{tabular}{llrc}
\hline Group & Parameters & ANNs & Synchrony \\
\hline Control & Median [mm] & 0.98 & 1.27 \\
& Interquartile range [mm] & 1.29 & 1.39 \\
\multirow{4}{*}{ Worst } & 95th percentile [mm] & 3.72 & 4.22 \\
& Median [mm] & 3.58 & 4.28 \\
& Interquartile range [mm] & 4.81 & 5.94 \\
& 95th percentile [mm] & 14.37 & 21.27 \\
\hline
\end{tabular}



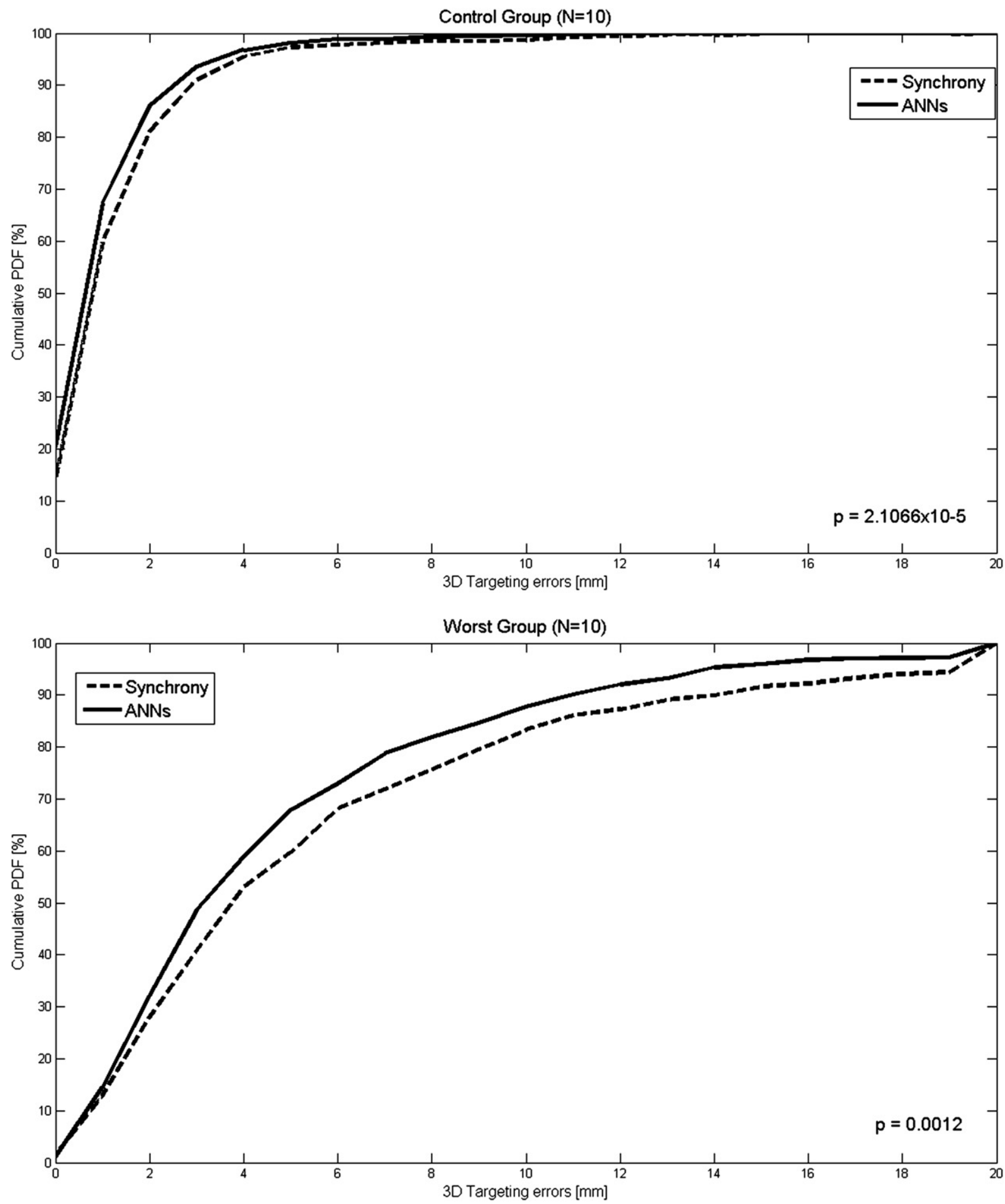

Figure 4 Cumulative probability distribution functions (PDFs) of 3D targeting errors for control (upper panel) and worst groups (lower panel).

Table 2 3D motion amplitude and period observed in the three acquisitions, quantified in terms of minimum, median and maximum values.

\begin{tabular}{llcc}
\hline & Minimum & Median & Maximum \\
\hline Motion Period [s] & 2.07 & 3.41 & 7.43 \\
3D Motion amplitude [mm] & 8.46 & 11.53 & 14.38 \\
\hline
\end{tabular}

target position as function of the position of three external markers. The proposed method was validated by quantifying 3D targeting errors in 20 cases selected from a large database of clinical dataset coming from patients treated with the CyberKnife system equipped with the Synchrony RTS module, whose performance was used as a benchmark. Although the analysis of Cyberknife data was already the subject of the reference [24], we decided to include it in 
Table 3 Statistical parameters of the 3D targeting errors recorded in three acquisitions on the moving phantom.

\begin{tabular}{lllr}
\hline $\begin{array}{l}\text { Acquisition/ } \\
\text { Parameters }\end{array}$ & $\begin{array}{l}\text { Median } \\
{[\mathrm{mm}]}\end{array}$ & $\begin{array}{l}\text { Interquartile } \\
\text { range }[\mathrm{mm}]\end{array}$ & $\begin{array}{l}\text { 95th percentile } \\
{[\mathrm{mm}]}\end{array}$ \\
\hline$\# 1$ & 1.03 & 0.99 & 2.75 \\
$\# 2$ & 1.01 & 0.72 & 10.72 \\
$\# 3$ & 1.35 & 0.66 & 2.15 \\
\hline
\end{tabular}

this study as a necessary validation step to prove the capabilities of the developed correlation algorithm before integrate it in the SMART-D system. Moreover, this analysis is no more aimed to compare different tumor tracking strategies and the algorithm presented in this work differs from those described in reference [24] since it is not meant to offline data analysis but to online data acquisition and processing. The analysis was focused on correlation errors, taking into account the accuracy in the correlation between external surrogate signals and inner tumor motion. The errors in time prediction, which is needed in order to compensate for system latencies, were not considered in this work. As a matter of fact, recent studies showed that prediction errors depend mainly on the latency period, whereas the main component in overall tumor tracking errors depends on the correlation model $[22,23]$.

Results obtained from both the control and the worst patient groups demonstrate that the developed algorithm, within the selected cases, yields an effective improvement in tracking accuracy with respect to Synchrony RTS. This results confirms the findings of the work in reference [24], where ANNs proved to be able to achieve better tracking accuracy if compared with the Synchrony RTS.

In particle therapy the requirement for an accurate dose conformation on the target volume is higher than in conventional treatments due to the increased geometrical selectivity and relative biological effectiveness that particles beams show with respect to photons [25]. For this reason, this kind of treatment is potentially more effective than a similar treatment delivered with photons. However, in order to exploit these advantages and avoid severe damage to healthy structures due to tumor miss, a greater accuracy in dose delivery is required. Therefore, as a consequence of its increased accuracy, the proposed method can be regarded as a starting point for the development of tumor tracking systems not only for photon radiotherapy but also for particle therapy.

The reported clinical data analysis also highlighted the need to implement online updating strategies for external/ internal correlation models. Fig. 6 shows how tracking errors, measured in correspondence to the acquired images, increased along the treatment time when neural networks were not retrained. Such a trend is not observed when the model was updated by retraining the neural networks with the first-in-first-out strategy described in the Methods section. This result was expected since the total treatment time for the considered cases is $72 \mathrm{~min}$ on average, with a maximum of $118.8 \mathrm{~min}$. It has been shown in the literature that internal/external correlation changes significantly over long time periods, thus making the estimation of target position unreliable if an updating strategy is not used.

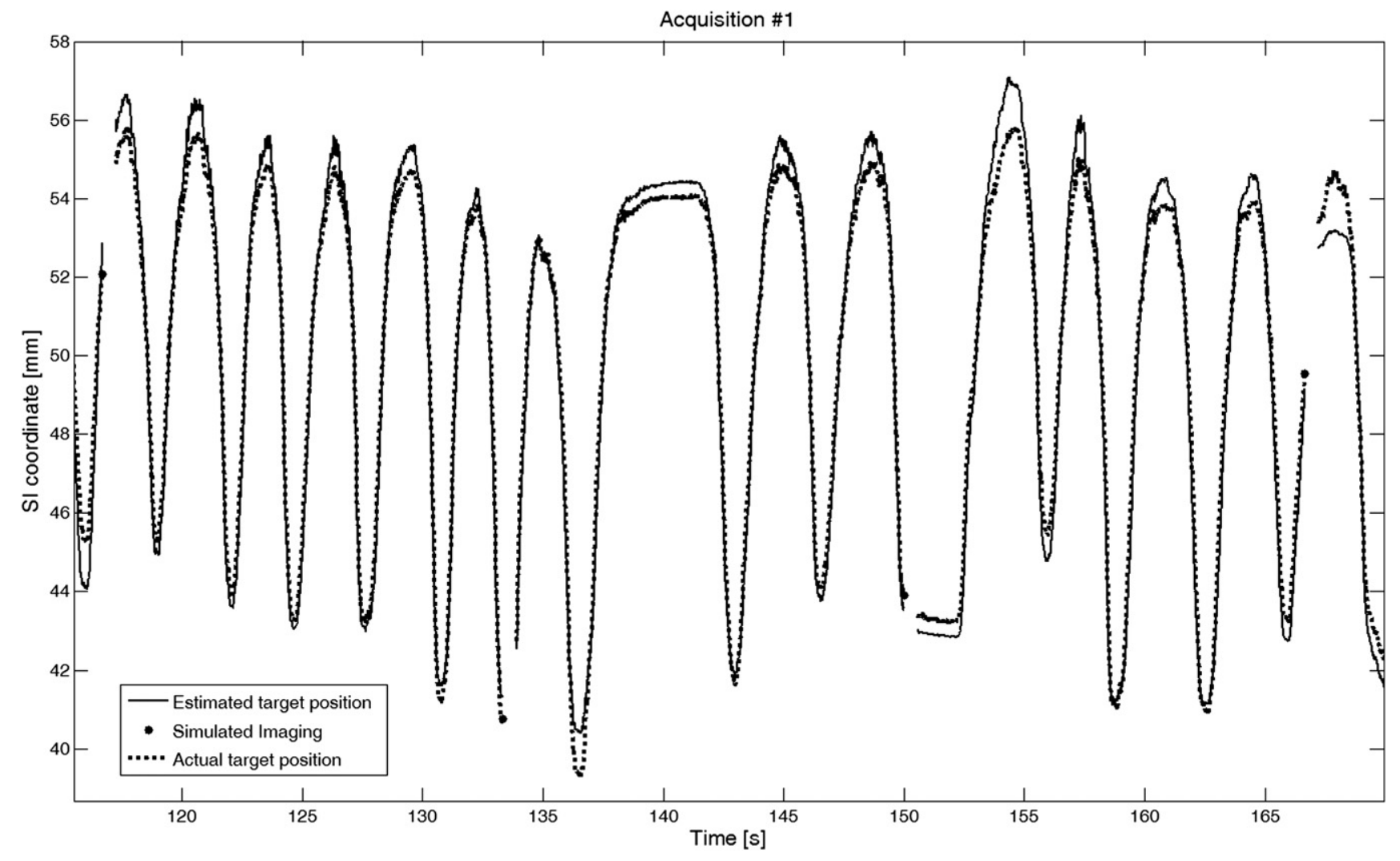

Figure 5 Exemplifying trace of the actual and estimated SI coordinate of the target, recorded in acquisition \#1. 


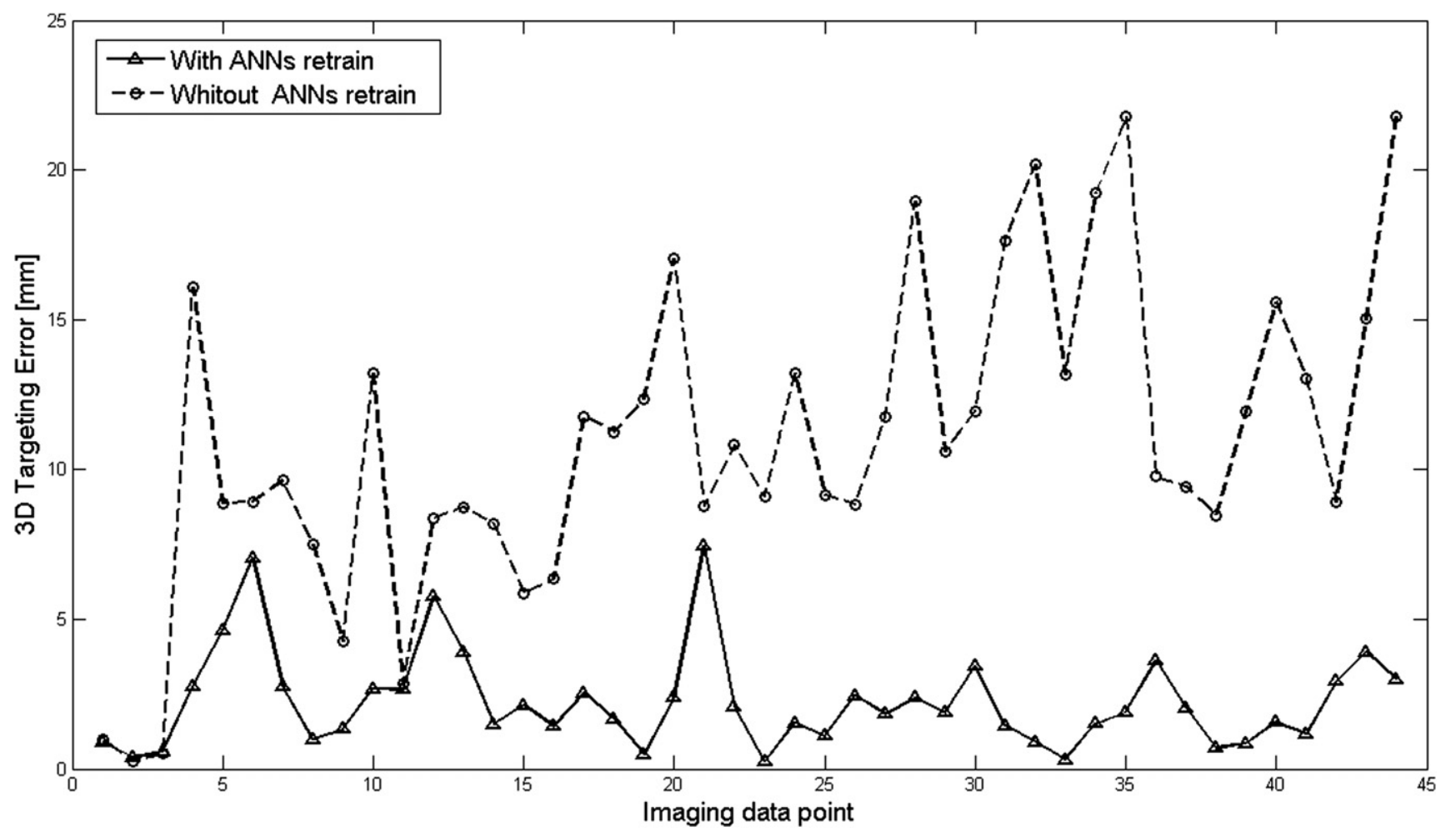

Figure 6 3DTEs obtained in a worst case with (solid line) and without (dotted line) neural networks retrain.

The ANN architecture was selected as a trade-off between tracking accuracy and computational time. Specifically, clinical application requires both real-time capabilities in tumor position estimation and acceptable workload for training and retraining procedures. We choose a double-layer architecture to include the complexity required to learn the internal/external correlation form Cyberknife data, which often exhibit baseline drifts as well as amplitude and frequency variations.

The hyperbolic tangent was selected as activation function so that possible peaks, caused by noise or heavy irregularities in the input data, are likely to marginally affect the output, due to the saturation of the activation function. In this way, improved output stability is expected. It should also be noted that a non-linear activation function is required, in order to include in the model the strong non-linearity that is typical in internal/ external correlation studies [28]. In this work, however, no specific effort was focused on the optimization of the ANN architecture. Considering the numerous parameters required to define the neural networks architecture, a dedicated study would most probably lead of an optimal solution, both in terms of network architecture and training algorithm, with potential improvement in tumor position estimation especially in presence of breathing irregularities.

We chose to implement three parallel neural networks instead of a single one with 9 inputs ( 3 coordinates for 3 markers). This approach replicates what is implemented in the Synchrony RTS, featuring an independent polynomial model for each of the external markers [20]. It also gives more flexibility to the retraining procedure, since each network can be treated independently from the others, thus providing a way to parallelize the retraining process. Alternative solutions to the simple average of the 3 independent target position estimations are feasible and would potentially increase the method robustness. For example, independent estimation could be averaged using as weights the current tracking errors measured for each network. In this case, however, the adequacy of the calculated weights along for the relatively extended period separating control image acquisition (on average $66.1 \mathrm{~s}$ for the 20 patients included in our study) should be thoroughly investigated. This requires the availability of a testing platform providing continuous information on external fiducials and target position.

We are planning to systematically investigate the use of a single neural network with 9 inputs instead of 3 neural network in future studies. However, first preliminary results show that the performance are not different.

In order to have a testing platform providing continuous information not only on the external fiducials but also on internal target position, the moving phantom for respiratory motion simulation was developed, thus providing the possibility to calculate the targeting errors at the same frequency of the external signals. Moreover, the moving phantom allowed us to validate the integration of the algorithm into the selected infra-red optical tracking system and to assess the capability to estimate tumor motion in real-time. Results obtained from the acquisitions performed on the moving phantom show that the developed algorithm is able to estimate the target position in realtime and can be integrated into a hardware platform dedicated to particle therapy, thus proving the feasibility of ANN-based tumor tracking strategies in this kind of treatments. 
Real-time tracking error calculation shows that accuracy is not influenced by respiratory frequency variation, as it can be observed in Fig. 5, where no phase shift are present. However, the same exemplifying figure shows that the accuracy reduction is concentrated in correspondence to the maximum expiration and inspiration peaks.

Despite the limited time duration of the experiments (no more than $3 \mathrm{~min}$ ) and the controlled conditions in which they were performed, retraining is still necessary to achieve acceptable accuracy. This could be caused by the limited generalization ability of the implemented neural networks, whose architecture was optimized on Cyberknife data. This latter may be too complex for signals acquired on the moving phantom and, as a consequence, it may lead to overfitting effects.

Technically, the actuation of the phantom can be a drawback as the motion of the pulley was obtained by the operator hand. However, the generated motion patterns were sufficiently repeatable thanks to the intrinsic mechanical constraints of the phantom. Since the moving phantom proved to be a useful tool for the validation of tumor tracking methods, we are planning a complete redesign to overcome its main limitations. For this reason, we envisage including electric actuation by means of a stepper motor, in order to obtain automated and precisely controlled movements, thus producing highly repeatable trajectories. Moreover we plan to replace wood with Polymethylmethacrylate (PMMA) as main construction material. In this way, friction between moving parts would be reduced, thus obtaining smoother movements. In addition, the remote electric control and the ability to operate the phantom both in standing and supine position, will allow to acquire dynamic tomographic images (4DCT), which could be exploited for additional validation of tumor tracking methods as well as for simulation of treatment planning data.

\section{Conclusion}

The study presented in this paper showed that artificial neural networks can be regarded as a valuable instrument for real-time tumor tracking, confirming the need for complex models to estimate tumor motion as a function of external surrogate signals.

Moreover, the integration of the developed algorithm into an infra-red optical tracking device for patient set-up verification in particle therapy has been proved to be feasible.

Further validation and optimization of the proposed method are needed, requiring a dataset where the ground truth target position is detected at higher frequency with respect to what is available in CyberKnife data and in general in clinical dataset. The described breathing phantom is put forward to represent a valuable platform in this respect, as it provides simultaneous real-time traces of target and fiducials, in controlled experimental conditions.

\section{References}

[1] Ross CS, Hussey DH, Pennington EC, Stanford W, Doornobs JF. Analysis of movement of intrathoracic neoplasms using ultrafast computerized tomography. Int J Radiat Oncol Biol Phys 1990;18:671-7.

[2] Langen KM, Jones DTL. Organ motion and its management. Int J Radiat Oncol Biol Phys 2001;50:265-78.

[3] Seco J, Sharp GC, Wu Z, Gierga D, Buettner F, Paganetti H. Dosimetric impact of motion in free-breathing and gated lung radiotherapy: a 4D Monte Carlo study of intrafraction and interfraction effects. Med Phys 2008;35:356-66.

[4] ICRU. Prescribing, recording, and reporting photon beam therapy. In: ICRU report, vol. 50. Bethesda: International Commission on Radiation Units and Measurements; 1993.

[5] ICRU. Prescribing, recording, and reporting photon beam therapy (supplement to ICRU report 50). In: ICRU report, vol. 62. Bethesda: International Commission on Radiation Units and Measurements; 1999.

[6] Baroni G, Garibaldi C, Riboldi M, Spadea MF, Catalano G, Tagaste B, et al. 3D optoelectronic analysis of interfractional patient setup variability in frameless extracranial stereotactic radiotherapy. Int J Radiat Oncol Biol Phys 2006;64:635-42.

[7] Gianoli C, Riboldi M, Spadea MF, Travaini LL, Ferrari M, Mei R, et al. A multiple points method for $4 \mathrm{D}$ CT image sorting. Med Phys 2011;38:656-67.

[8] Meeks SL, Tomé WA, Willoughby TR, Kupelian PA, Wagner TH, Buatti JM, Bova FJ. Optically guided patient positioning techniques. Semin Radiat Oncol 2005;15:192-201.

[9] Ford E, Mageras G, Yorke E, Rosenzweig KE, Wagman R, Ling CC. Evaluation of respiratory movement during gated radiotherapy using film and electronic portal imaging. Int $\mathrm{J}$ Radiat Oncol Biol Phys 2002;52:522-31.

[10] Bert C, Metheany KG, Doppke K, Chen GT. A phantom evaluation of a stereo-vision surface imaging system for radiotherapy patient setup. Med Phys 2005;32:2753-62.

[11] Seppenwoolde Y, Shirato H, Kitamura K, Shimizu S, Van Herk M, Lebesque JV, Miyasaka K. Precise and real-time measurement of $3 \mathrm{D}$ tumor motion in lung due to breathing and heartbeat, measured during radiotherapy. Int J Radiat Oncol Biol Phys 2002;53:822-34.

[12] Shirato H, Shimizu S, Shimizu T, Nishioka T, Miyasaka K. Realtime tumor-tracking radiotherapy. Lancet 1999;353. 1331-31.

[13] Shirato H, Shimizu S, Kunieda T, Kitamura K, Kagei K, Nishioka $T$, et al. Physical aspects of a real-time tumortracking system for gated radiotherapy. Int J Radiat Oncol Biol Phys 2000;48:1187-95.

[14] Keall PJ, Mageras GS, Balter JM, Emery RS, Forster KM, Jiang $S B$, et al. The management of respiratory motion in radiation oncology report of AAPM Task Group 76. Med Phys 2006;33:3874-900.

[15] Wong J, Sharpe M, Jaffray D, Kini VR, Robertson JM, Stromberg JS, et al. The use of active breathing control (ABC) to minimize breathing motion during radiation therapy. Int $J$ Radiat Oncol Biol Phys 1999;44:911-9.

[16] Hanley J, Debois MM, Mah D, Mageras GS, Raben A, Rosenzweig K, et al. Deep inspiration breath-hold technique for lung tumors: the potential value of target immobilization and reduced lung density in dose escalation. Int J Radiat Oncol Biol Phys 1999;45:603-11.

[17] Mah D, Hanley J, Rosenzweig KE, Yorke E, Bradan L, Ling CC, et al. Technical aspects of the deep inspiration breath-hold technique in the treatment of thoracic cancer. Int J Radiat Oncol Biol Phys 2000;48:1175-85.

[18] Ohara K, Okumura T, Akisada M, Inada T, Mori T, Yokota H, et al. Irradiation synchronized with respiration gate. Int $\mathrm{J}$ Radiat Oncol Biol Phys 1989;17:853-7.

[19] Kubo HD, Hill BC. Respiration gated radiotherapy treatment: a technical study. Phys Med Biol 1996;41:83-91.

[20] Kilby W, Dooley JR, Kuduvalli G, Sayeh S, Maurer Jr CR. The CyberKnife robotic Radiosurgery system in 2010. Technol Cancer Res Treat 2010;9:433-52. 
[21] Schweikard A, Glosser G, Bodduluri M, Murphy MJ, Adler JR. Robotic motion compensation for respiratory movement during Radiosurgery. Comput Aided Surg 2000;5: 263-77.

[22] Seppenwoolde Y, Berbeco RI, Nishioka S, Shirato H, Heijmen B. Accuracy of tumor motion compensation algorithm from a robotic respiratory tracking system: a simulation study. Med Phys 2007;34:2774-84.

[23] Hoogeman MJ, Prevost B, Nuyttens J, Poll J, Levendag P, Heumen B. Clinical accuracy of the respiratory tumor tracking system of the cyberknife: assessment by analysis of log files. Int J Radiat Oncol Biol Phys 2009;74:297-303.

[24] Torshabi AE, Pella A, Riboldi M, Baroni G. Targeting accuracy in real-time tumor tracking via external surrogates: a comparative study. Technol Cancer Res Treat 2010;9: 551-62.

[25] Kraft G. Tumor therapy with heavy charged particles. Prog Part Nucl Phys 2000;45:S473-544.

[26] Grözinger SO, Bert C, Haberer T, Kraft Rietzel E. Motion compensation with a scanned ion beam: a technical feasibility study. Radiat Oncol 2008;3:34.

[27] Bert C, Gemmel A, Saito N, Chaudhri N, Schardt D, Durante M, et al. Dosimetric precision of an ion beam tracking system. Radiat Oncol 2010;5:61.

[28] Ruan D, Fessler JA, Balter JM, Berbeco RI, Nishioka S, Shirato $\mathrm{H}$. Inference of hysteretic respiratory tumor motion from external surrogates: a state augmentation approach. Phys Med Biol 2008;53:2923-36. 